\title{
e-Health: Data Integration, Data Mining, and Knowledge Management in Health Informatics
}

\author{
Yanchun Zhang \\ Centre for Applied Informatics \& School of Engineering and Science \\ Victoria University, Australia \\ yanchun. zhang@vu.edu.au
}

\begin{abstract}
Keynote Speech. In last few decades, with the advent of database systems and networking technologies, a huge volume of health data and valuable medical knowledge have been electronically available, accessible and processible, especially over the virtual cyberspace - the Web, even from a remote corner in the world. Nowadays the wide deployment of Hospital Information Management Systems (HIMS) and Web based clinical or medical systems, for example, the Medical Director, a generic GP clinical system, have made it possible to record, disseminate and implement the health information and clinical practices easily and globally. And health care and medical service is becoming more data-intensive and evidence-based since electronic health records are used to track individuals' and communities' health information (particularly changes). These substantially motivate and advance the emergence and the progress of data-centric health data and knowledge management research and practice, for example, Health Informatics.

In this presentation, we will introduce several case studies and research projects to address the challenges encountered in health service. These include data exchange and health service Integration in health information systems, health information visualisation, data mining and data analyse for patient care management. We will then introduce a framework of data integration, knowledge management and user behaviour modelling for complementing and improving existing health care and service systems.
\end{abstract}

